\section{Long-term real-world results of ibrutinib therapy in patients with relapsed or refractory chronic lymphocytic leukemia: 30-month follow up of the Swedish compassionate use cohort}

Ibrutinib is standard of care treatment for patients with chemoimmunotherapy-refractory chronic lymphocytic leukemia (CLL). We have previously reported the early (median follow up 10 months) real-world results on ibrutinib in strictly consecutive Swedish CLL patients treated in a compassionate use program (CUP). ${ }^{1}$ We report here the 30-month follow up on progression-free survival (PFS), overall survival (OS), and safety.

The CUP was open for inclusion between May 15, 2014 and May 31, 2015. The program offered free drug access for patients with CLL until ibrutinib was generally available on the market. All patients included in the CUP were identified and this retrospective study was conducted at 27 Swedish hospitals. Data were extracted from each patient's medical file and entered into case record forms (CRF) by the treating physician. Monitoring of data from individual patient files was performed by the academic study team and cross-checked with the CRFs. The procedure was approved by the regional ethics committee (www.epn.se) and conducted in accordance with the Declaration of Helsinki.

Eligibility criteria for the CUP have been described earlier. In brief, patients had a confirmed diagnosis of CLL or small lymphocytic lymphoma (SLL) according to International Workshop on Chronic Lymphocytic Leukemia (IWCLL) criteria, ${ }^{2}$ had high-risk disease that did not respond to chemoimmunotherapy or that progressed within 24 months or had $\operatorname{del}(17 \mathrm{p})$ or TP53 mutation. Inclusion criteria also included: Performance Status (PS) $\leq 2$, neutrophil count $\geq 0.5 \times 10^{9} / \mathrm{L}$, and platelet count $\geq 30 \times 10^{9} / \mathrm{L}$. Patients received $420 \mathrm{mg}$ oral ibrutinib once daily until progression or occurrence of unacceptable side effects. Individual dose modifications were at the discretion of the treating physician. End points were overall response rate (ORR), PFS, OS, and safety, as previously defined. ${ }^{1}$ The impact of dose reductions and treatment breaks on PFS and OS was analyzed. In addition, we studied the impact of inclusion/exclusion criteria (obtained from the RESONATE trial ${ }^{3}$ ) on the outcome of patients treated in the Swedish CUP.

Cumulative Index Rating Scale (CIRS) was used to define comorbidities at baseline. ${ }^{4}$ Treatment toxicity was evaluated using National Cancer Institute Common Terminology Criteria for Adverse Events v.3.0, except for anemia, thrombocytopenia and neutropenia, which were graded in accordance with the IWCLL grading scale. ${ }^{2}$ Adverse events (AE) grade 3 or higher were recorded. PFS and OS were estimated by the Kaplan-Meier method. OS was defined as time from start of ibrutinib treatment to death or latest follow up. PFS was defined as time from start of treatment to progression, start of new anti-cancer treatment, or death from any cause. Four patients proceeded to allogeneic stem cell transplantation (allo-SCT) and two patients had donor lymphocyte infusion (DLI) after having responded to ibrutinib; these six patients were censored from the PFS analysis at the time of alloSCT or DLI. Univariate and multivariate analyses on time to failure were estimated using the Cox proportional regression hazards model. Statistical analyses were performed using Stata 14.2 (Stata Statistical Software; StataCorp LP, College Station, TX, USA). Ninety-five patients were included in the study. Median age was 69 years, 63\% had del(17p)/TP53 mutation, 27\% had PS
Table 1. Adverse events grade $3-4{ }^{1}$

\begin{tabular}{lc}
\hline Hematologic AEs & Grade $3-4(\%)$ \\
Anemia $n=91$ & 0 \\
Thrombocytopenia $^{2} \mathrm{n}=87$ & 20 \\
\hline Neutropenia $^{3} \mathrm{n}=79$ & 41 \\
Non-hematologic AEs excluding infections & \\
Arthralgia & 4 \\
Congestive heart failure & 3 \\
Exanthema & 2 \\
Fracture & 3 \\
Hypertension & 2 \\
Hyperviscosity syndrome & 4 \\
Other $^{5}$ & 2 \\
Inections & 16
\end{tabular}

Infections

Pneumonia

Febrile neutropenia or septicemia

13

Other

31

Expressed as percentage of patients. ${ }^{2}$ In case of platelet count $<20 \times 10^{\%} / \mathrm{L}$ before therapy the patient was not evaluable for toxicity, according to the International Workshop on Chronic Lymphocytic Leukemia (IWCLL) criteria. ${ }^{3}$ In case of absolute neutrophil count $<1 \times 10^{9} / \mathrm{L}$ before therapy the patient was not evaluable for toxicity, according to IWCLL criteria. ${ }^{4}$ Patients with absolute lymphocyte count 899 and $917 \times 10^{9} / \mathrm{L}$, respectively. Resolved following leukapheresis. ${ }^{5}$ Total of 15 patients, including: acute myocardial infarction $n=1$, atrial fibrillation $n=1$, cutaneous ulcers $n=1$, choking $n=1$, diarrhea $n=1$, hemorrhage $n=1$, pleural fluid $n=1$, psychiatric disorder $n=1$, portal thrombosis $n=1$, pulmonary embolism $n=1$, renal failure $n=1$, renal carcinoma $n=1$, subileus $n=1$, trauma $n=1$, unilateral blindness due to macula degeneration $n=1$. AE: adverse event.

grade $2-3$, and the median number of prior therapies was 3. The ORR was $84 \%$; complete remission (CR) rate was not evaluable since a bone marrow examination was carried out in only a few of the responding patients. Median ibrutinib treatment duration was 27 months (range 0.638 ) and $51 \%$ remained on ibrutinib therapy at follow up. At a median follow-up time of 30 months (range 1-38), the PFS rate was $52 \%$ and the OS rate was $63 \%$ (Figure $1 \mathrm{~A}$ and $\mathrm{B})$. Next, we analyzed the impact of the inclusion/exclusion criteria obtained from the pivotal RESONATE study: $44 \%$ of CUP patients had at least one exclusion criteria, the most common being PS $(n=22)$, previous malignancy $(\mathrm{n}=11)$, neutropenia $(\mathrm{n}=7)$, and thrombocytopenia $(n=3)$, confirming our cohort to be representative of real-world situations. OS was significantly $(P<0.05)$ shorter for CUP patients not matching the RESONATE inclusion criteria. However, there was no significant difference in PFS (Figure $1 \mathrm{C}$ and D); this may be explained mainly by the age and worse performance status in our patients versus those in the RESONATE study. In contrast to our early (10-month) report, ${ }^{1}$ the negative survival impact of $\operatorname{del}(17 \mathrm{p}) / T P 53$ mutation was no longer significant, but patients with $\operatorname{del}(17 \mathrm{p}) / T P 53$ mutation and $\geq 3$ previous therapies $(n=25)$ had a shorter OS compared to patients with $0-2$ previous therapies (median 20.7 months $v s$. not reached; $P<0.01$ ). In multivariate analyses, OS was significantly associated with baseline comorbidities (CIRS), and PFS was associated with CIRS and number of prior therapies.

Fifty-one percent of patients had a grade 3-4 infection (Table 1): $22 \%$ pneumonia, $13 \%$ febrile neutropenia/septicemia, and $31 \%$ other infections. Thirteen percent had grade 3-5 opportunistic infections. Forty-one percent and $20 \%$ had grade 3-4 neutropenia or thrombocytopenia, respectively. Richter transformation (RT) occurred in 12 
patients $(13 \%)$ after a median time of 14 months (range 4-36); a clear-cut plateau of RT incidence has not yet been observed. The incidence is slightly higher than in most other clinical trial reports and is probably due to the fact that our patients had more advanced disease than patients recruited into the clinical trials. In addition, we up-dated the cumulative incidence of atrial fibrillation (all
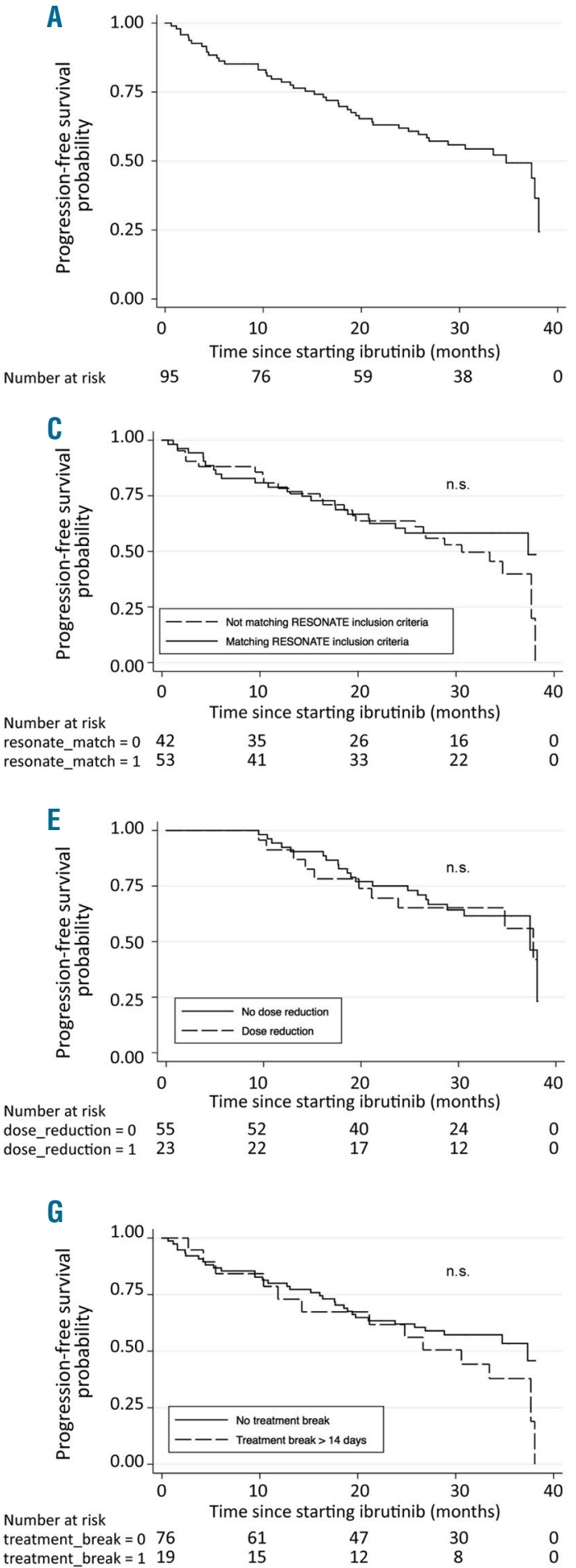

grades). Fifteen percent of our patients developed atrial fibrillation during ibrutinib therapy. This is in comparison with the cumulative incidence of atrial fibrillation of $8 \%$ at 10 months follow up, ${ }^{1}$ implying that the risk of atrial fibrillation is substantial during long-term treatment. Thirty-seven percent of the patients had died due to: RT $(n=12)$, infection $(n=7)$, CLL progression $(n=6)$, second
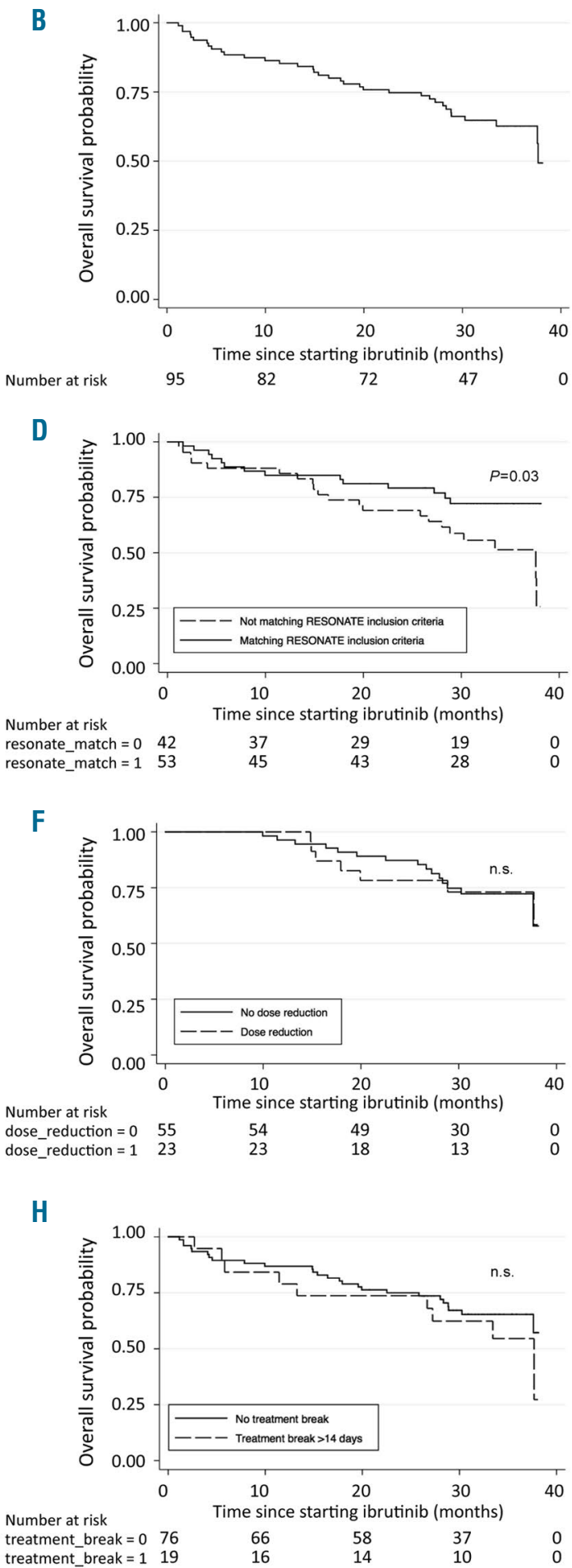

Figure 1. Progression-free survival (PFS) and overall survival (OS) for patients included in the compassionate use program (CUP). Survival analyses from start of treatment until event using the Kaplan-Meier method. (A) PFS and (B) OS of all patients. (C) PFS and (D) OS of CUP patients matching versus not matching the RESONATE trial inclusion criteria. (E) PFS and (F) OS of CUP patients with dose reduction versus no dose reduction. (G) PFS and (H) OS of CUP patients with treatment break versus no treatment break. 
malignancy $(n=5)$, miscellaneous toxicity $(n=4)$, and sudden death $(n=1)$. Ibrutinib was permanently stopped in 47 patients $(49 \%)$. The reasons for treatment discontinuation were: toxicity $(n=19)$, RT $(n=11)$, CLL progression $(n=6)$, allo-SCT $(n=4)$, second malignancy $(n=3)$, need of dual antiplatelet therapy $(n=3)$, and sudden death $(n=1)$. Toxicities leading to treatment discontinuation were: infection $(n=8)$, generalized exanthema or blisters $(n=3)$, bleeding $(n=1)$, anxiety $(n=1)$, elevated liver enzymes $(n=1)$, thrombocytopenia $(n=1)$, neutropenia $(n=1)$, diarrhea $(n=1)$, subdural hematoma $(n=1)$, and myelodysplastic syndrome $(n=1)$. Six patients were switched to treatment with idelalisib, due to the need for dual antiplatelet therapy $(n=3)$, CLL progression $(n=2)$, and skin toxicity $(n=1)$. At the time of this follow up, no patient had yet received venetoclax. The discontinuation rate of $49 \%$ at 30 months follow up in our study contrasts somewhat to the $42 \%$ discontinuation rate already seen at a 17 -month follow up in the recently reported real-world report by Mato et al. ${ }^{5}$ Twenty-five patients had dose reduction and/or treatment breaks with a cumulative length of more than 4 months. Thirteen patients had dose reductions to $280 \mathrm{mg}, 10$ patients to $140 \mathrm{mg}$, and 2 patients had treatment withheld for more than 4 months. Next, the impact of dose reductions on PFS and OS was analyzed. Patients with a treatment break $>4$ months $(n=2)$ or treatment length $<8$ months $(n=15)$ were excluded. Dose reductions did not have any significant impact on PFS or OS (Figure 1E and F) confirming the findings by Mato et al. ${ }^{6}$ Similarly, no impact of dose reduction on PFS/OS was observed if all patients were included, i.e. also those with treatment break $>4$ months and/or treatment length $<8$ months. Nineteen patients had treatment breaks $>14$ days (median 22 days). In contrast to others, ${ }^{7}$ we found no significant impact of such treatment breaks on PFS or OS (Figure $1 \mathrm{G}$ and H). Four of 6 patients who progressed while on ibrutinib were tested for Bruton's tyrosine kinase (BTK) mutation at disease progression; all carried a $C 481 S$ mutation in $>50 \%$ of the cells (Sanger sequencing). Cryopreserved CLL cells from two of these patients were tested for BTK mutation at baseline and both were negative.

To our knowledge, this is the first report on long-term follow up of ibrutinib therapy in a real-world setting in consecutive, well-defined patients with advanced phase CLL. Nearly half of the patients had stopped ibrutinib treatment at 30 months, which, as expected, is higher than what was reported at the same time point in the 3year follow up of the first clinical trial of ibrutinib. ${ }^{8}$ Early treatment discontinuation due to toxicity in this study was less common than in the recently reported realworld analysis by Mato et al. ${ }^{5}$ The reason for this difference is not clear. Dose reductions were common in the Swedish cohort but did not impact on the long-term benefit of ibrutinib. We conclude that ibrutinib is an effective and well-tolerated treatment for long-term use in a wider patient population. Nevertheless, disease progression or non-tolerability represent continuous events, and the need for new effective treatment regimens in CLL is still essential. In the new era of high-cost medications, carefully conducted real-world studies add important information to that provided by clinical trials.

Maria Winquist, ${ }^{1,2}$ Per-Ola Andersson, ${ }^{3}$ Anna Asklid, ${ }^{2}$

Karin Karlsson, ${ }^{4}$ Claes Karlsson, ${ }^{1,2}$ Birgitta Lauri, ${ }^{5}$

Jeanette Lundin, ${ }^{1,2}$ Mattias Mattsson, ${ }^{6}$ Stefan Norin, ${ }^{7}$

Anna Sandstedt, ${ }^{8}$ Richard Rosenquist, ${ }^{9}$ Florentin Späth, ${ }^{10}$

Lotta Hansson ${ }^{1,2}$ and Anders Österborg; ${ }^{1,2}$ for the Swedish CLL Group

${ }^{1}$ Department of Hematology, Karolinska University Hospital, Stockholm; '2Department of Oncology-Pathology, Karolinska Institutet, Stockholm; ${ }^{3}$ Department of Hematology, Borås Hospital; ${ }^{4}$ Department of Hematology, Lund University Hospital; ${ }^{5}$ Department of Hematology, Sunderby Hospital, Sunderbyn-Lulea; ${ }^{6}$ Department of Hematology, Uppsala University Hospital; 'Department of Internal Medicine, Karolinska Institutet, Stockholm; ${ }^{8}$ Department of Hematology, Linköping University Hospital; 'Department of Molecular Medicine and Surgery, Karolinska Institutet, Stockholm and ${ }^{10}$ Department of Radiation Sciences, Oncology, Umeå University, Sweden

Correspondence: MARIA WINQVIST. maria.winqvist@sll.se doi:10.3324/haematol.2018.198820

Part of this research was presented at the 23rd Congress of the European Hematology Association (EHA), June 14-17, 2018, Stockholm, Sweden

Information on authorship, contributions, and financial \& other disclosures was provided by the authors and is available with the online version of this article at www. haematologica.org.

\section{References}

1. Winqvist M, Asklid A, Andersson PO, et al. Real-world results of ibrutinib in patients with relapsed or refractory chronic lymphocytic leukemia: data from 95 consecutive patients treated in a compassionate use program. A study from the Swedish Chronic Lymphocytic Leukemia Group. Haematologica. 2016;101(12):1573-1580.

2. Hallek M, Cheson BD, Catovsky D, et al. Guidelines for the diagnosis and treatment of chronic lymphocytic leukemia: a report from the International Workshop on Chronic Lymphocytic Leukemia updating the National Cancer Institute-Working Group 1996 guidelines. Blood. 2008;111(12):5446-5456.

3. Byrd JC, Brown JR, O'Brien S, et al. Ibrutinib versus ofatumumab in previously treated chronic lymphoid leukemia. N Engl J Med. 2014;371(3):213-223.

4. Goede V, Bahlo J, Chataline V, et al. Evaluation of geriatric assessment in patients with chronic lymphocytic leukemia: Results of the CLL9 trial of the German CLL study group. Leuk Lymphoma. 2016;57(4):789-796.

5. Mato AR, Nabhan C, Thompson MC, et al. Toxicities and outcomes of 616 ibrutinib-treated patients in the United States: a real-world analysis. Haematologica. 2018;103(5):874-879.

6. Mato AR, Timlin C, Ujjani C, et al. Comparable outcomes in chronic lymphocytic leukaemia (CLL) patients treated with reduced-dose ibrutinib: results from a multi-centre study. Br J Haematol. 2018;181(2):259-261.

7. UK CLL Forum. Ibrutinib for relapsed/refractory chronic lymphocytic leukemia: a UK and Ireland analysis of outcomes in 315 patients. Haematologica. 2016;101(12):1563-1572.

8. Byrd JC, Furman RR, Coutre SE, et al. Three-year follow-up of treatment-naive and previously treated patients with CLL and SLL receiving single-agent ibrutinib. Blood. 2015;125(16):2497-2506. 